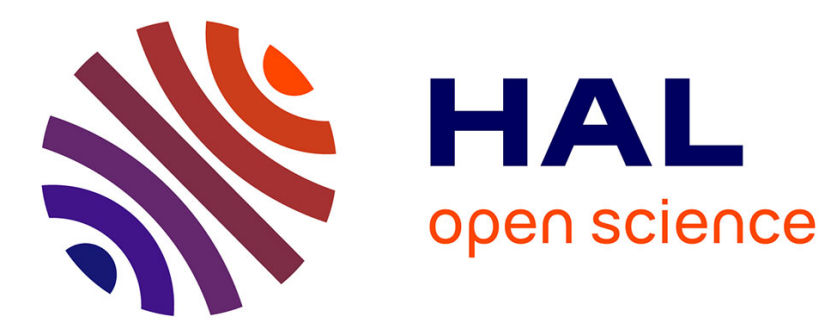

\title{
Broken symmetry of in-gap quasiparticle excitations in superconducting FeSe(001) with high defect density
}

Jonathan Baptista, Sergio Vlaic, Pierre Toulemonde, Sandra Karlsson, Pierre

Strobel, Dimitri V Roditchev, Stéphane Pons

\section{- To cite this version:}

Jonathan Baptista, Sergio Vlaic, Pierre Toulemonde, Sandra Karlsson, Pierre Strobel, et al.. Broken symmetry of in-gap quasiparticle excitations in superconducting FeSe(001) with high defect density. Physical Review B, 2018, 98 (22), pp.220502(R). 10.1103/PhysRevB.98.220502 . hal-01996196

\section{HAL Id: hal-01996196 \\ https://hal.science/hal-01996196}

Submitted on 8 Oct 2021

HAL is a multi-disciplinary open access archive for the deposit and dissemination of scientific research documents, whether they are published or not. The documents may come from teaching and research institutions in France or abroad, or from public or private research centers.
L'archive ouverte pluridisciplinaire HAL, est destinée au dépôt et à la diffusion de documents scientifiques de niveau recherche, publiés ou non, émanant des établissements d'enseignement et de recherche français ou étrangers, des laboratoires publics ou privés. 


\title{
Broken symmetry of in-gap quasiparticle excitations in superconducting $\mathrm{FeSe}(001)$ with high defect density
}

\author{
Jonathan Baptista, ${ }^{1,2,3}$ Sergio Vlaic, ${ }^{1,2,3}$ Pierre Toulemonde, ${ }^{4}$ Sandra Karlsson, ${ }^{4}$ Pierre Strobel, ${ }^{4}$ \\ Dimitri Roditchev, ${ }^{1,2,3,5}$ and Stéphane Pons ${ }^{1,2,3, *}$ \\ ${ }^{1}$ Laboratoire de Physique et d'Études des Matériaux, École de Physique et Chimie Industrielles de la Ville de Paris, ESPCI Paris, \\ PSL Research University, Paris, France \\ ${ }^{2}$ Sorbonne Université, UPMC University, Paris, France \\ ${ }^{3}$ CNRS UMR8213, Paris, France \\ ${ }^{4}$ Université Grenoble Alpes, CNRS, Grenoble INP, Institut Néel, F-38000 Grenoble, France \\ ${ }^{5}$ Institut des Nanosciences de Paris, Université Pierre et Marie Curie (UPMC), CNRS UMR7588, Paris, France
}

(Received 30 August 2018; revised manuscript received 16 November 2018; published 4 December 2018)

\begin{abstract}
FeSe(001) with a high defect density was studied in the superconductiong phase by means of scanning tunneling spectroscopy at low temperature. Quasiparticle excitations surrounding structural defects are found inside the superconducting gap. This spectral intensity is used for revealing additional scattering channels for the quasiparticle excitations. The related wave vectors are located around the $M$ points of the Brillouin zone and follow $C_{2}$ symmetry. We attribute the symmetry and the observed lack of dispersion of the scattering channels to the interaction of Bogoliubov quasiparticles with nematic order.
\end{abstract}

DOI: 10.1103/PhysRevB.98.220502

The origin of the nematicity $[1,2]$ of the electronic properties of the family members of iron superconductors at low temperatures [3-7] is still not understood. On the one hand, it could be solely related to the structural transition from tetragonal to orthorhombic symmetry and provides the breaking of rotational symmetry, but the structural transition itself could be a side effect of this nematicity. On the other hand, many additional experimental and theoretical works suggest that the nematicity could be due to other electronic instabilities such as magnetic fluctuations or orbital order [8-15]. Among the iron-based superconductors, $\mathrm{FeSe}$ is a compound with the simplest crystal structure consisting of FeSe planes separated in the [001] crystallographic direction by van der Waals gaps [16]. In FeSe, the nematic order seems to be intimately linked to the structural transition-which takes place at $T_{S} \approx 90 \mathrm{~K}$-in such a way that the electronic structure of the detwinned crystal determined by angle-resolved photoemission spectroscopy (ARPES) presents a $C_{2}$ symmetry [17]. The electronic structure of bulk FeSe crystals is composed of spin-orbit split multiple bands with significant correlations at the Fermi level [12,17-23]. The Fermi surface originates from the iron $3 d$ orbitals with a low Fermi energy and consists of disconnected two-dimensional hole and electron pockets centered at the $\Gamma$ and $M$ points, respectively. Spin and orbital momenta are entangled by spin-orbit interactions and thus structural, orbital order, and spin fluctuations could be mutually involved in the emergence of the nematicity. The material has no magnetic static order at low temperatures but it is very close to being magnetic [24-26], and bosonic modes associated with spin excitations were evidenced by means of inelastic neutron scattering [14,15]. It is generally

\footnotetext{
*stephane.pons@espci.fr
}

thought that superconductivity is not driven by conventional electron-phonon interactions because the superconducting order parameter, although not yet definitely determined, is not of a standard isotropic $s$ symmetry. Also, the way superconductivity interacts with the nematic order is not clear $[11,14]$. Conventional superconductors are characterized by a full gap at the Fermi level in the quasiparticle excitation spectrum and quasiparticle scattering at magnetic impurities induces a gap filling until superconductivity is destroyed. Iron-based superconductors are peculiar because they are made of magnetic elements at concentrations up to $50 \%$ which do not annihilate superconductivity. They also show significant quasiparticle spectral weight inside the pairing gap. In this Rapid Communication, we aim to elucidate the origin of the quasiparticle excitations in the superconducting gap of $\mathrm{FeSe}(001)$ by means of scanning tunneling microscopy and spectroscopy (STM/STS) at low temperature $(1.3 \mathrm{~K})$ in ultrahigh vacuum.

The studied samples- $\mathrm{FeSe}(001)$ single crystals-were cleaved under ultrahigh vacuum at room temperature before introduction in the STM held at $1.3 \mathrm{~K}$ under ultrahigh vacuum $\left(1 \times 10^{-10}\right.$ mbar $) \cdot \frac{d I}{d V}(x, y)$ conductance images were obtained by numerical differentiation of $I(V)$ spectra taken at a constant height (feedback off) at each point of an STM image (feedback on). This experimental mode allows for discriminating possible topographic effects [27] from spectroscopic effects [28]. Bias voltages are applied to the sample with respect to the grounded tip. Samples of the same batch were characterized elsewhere by various other experimental techniques [29-31]. The critical superconducting temperature of our sample was measured to be slightly below $8 \mathrm{~K}$, which was chosen on purpose to be weaker than our best crystals. This rather low temperature is explained by the large density of structural defects of various natures, as visible in the typical 

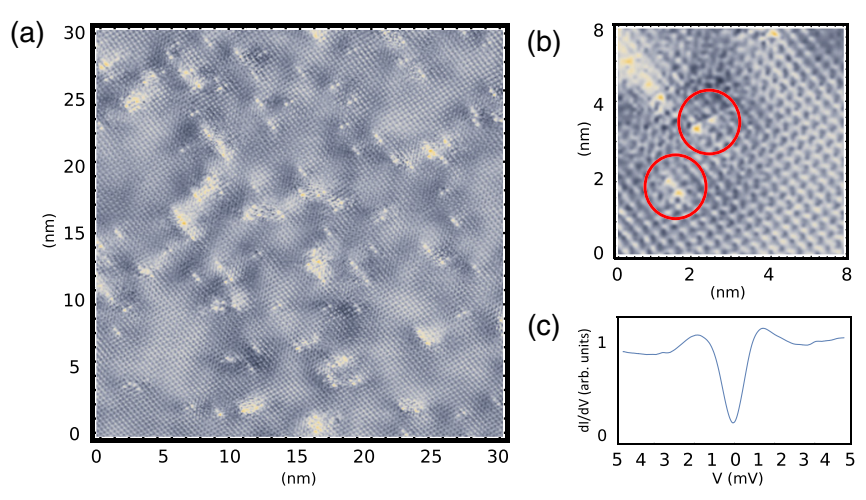

FIG. 1. (a) Topographic STM image of FeSe(001) at atomic resolution showing a high density of structural defects. (b) Zoom on typical defects. (c) Average scanning tunneling spectrum. The superconducting gap shows many peculiar features: It has an overall $\mathrm{V}$ shape with nonzero conductance at the Fermi level and the quasiparticle peaks are damped. Stabilization parameters: $I=600$ pA, $V=8 \mathrm{mV}$.

microscopy image presented in Fig. 1. The atomic corrugation of the topography image corresponds to the position of the topmost Se atoms [32]. STM is not accurate enough to evidence the small $(<0.3 \%)$ orthorhombic distortion of the surface and reveals a rather square symmetry. Thus, the topographic image is compatible with a unit cell composed of two $\mathrm{Fe}$ atoms with an apparent $C_{4}$ symmetry. A large amount of defects was commonly found as shown in Fig. 1(b): According to Ref. [33], the ones with two sharp spots can be ascribed to Fe vacancies while others manifest themselves as a weak corrugation of the surface and are of unknown origin. The topographical signature of Fe vacancies is oriented with respect to the orthorhombic compression (along the $\boldsymbol{a}$ or $\boldsymbol{b}$ axis). The high quantity of these defects oriented in two different directions implies that our sample was composed of a patchwork of small orientational domains separated by twin boundaries, yet one of the two orientations is clearly dominant in Fig. 1(a).

The average gap amplitude as estimated in Fig. 1 is around $2.2 \mathrm{meV}$. This is a rather low amplitude with respect to other works made with crystals with lower defect densities [10,34]. This spectrum exhibits a peculiar $\mathrm{V}$ shape and the quasiparticle peaks are almost washed out. The Fermi energy is low and this fact puts iron selenide in the category of superconductors close to the BEC-BCS transition [35]. The origin of an unusually strong gap filling and the typical $\mathrm{V}$-shaped spectral background could rely either on the specificity of the superconducting mechanism (e.g., anisotropic order parameter or multiband superconductivity with nonequivalent gaps) or in any process that perturbs the pair condensate and breaks the Cooper pairs. In the following, we use the interaction of the Cooper pairs with defects to explore the nature of the in-gap quasiparticle excitations.

Most of the defects, including point defects, seem not to interact strongly with superconductivity and preserve the superconducting gap in their vicinity. However, conductance maps at zero bias (ZB) reveal peculiar large defects showing a sharp resonance at the Fermi level which overcomes the superconducting gap, as seen in Figs. 2(a)-2(f). The analysis of the amplitude of the zero-bias conductance as a function of distance shows that the superconducting properties are recovered at typical distances of $5.5 \AA$ from these very localized strong defects. Thus, these defects behave as YuShiba-Rusinov impurities in a standard superconductor, i.e., they break locally the Cooper pairs, and their spatial influence is quite isotropic over the distance of the coherence length of the Cooper pairs [36] [of the order of a nm in FeSe(001)]. The density of these defects is still too low to produce a percolating effect. However, an additional smoother interacting potential makes the zero-bias spectral intensity spatially inhomogeneous, as seen in Fig. 2(g). The nature of this smooth underlying perturbation is unknown but it provokes an additional moderate gap filling without breaking superconductivity. This supplementary spectral intensity in the superconducting gap opens the opportunity to probe efficiently the symmetry of the scattering channels of the Bogoliubov quasiparticles in FeSe.

The Fourier transform of the conductance images for energies below and above the superconducting gap are presented in Fig. 3. These images allow us to determine the relevant wave vectors of the interference patterns of coherent quasiparticles or Bogoliubov quasiparticles. Out of the superconducting gap, at $\pm 2 \mathrm{meV}$, elliptical spots are located at the centers of the Brillouin zones (BZs) around the $\Gamma_{n, m}$ points given by the reciprocal lattice vectors $\boldsymbol{G}^{(n, m)}=(2 n \pi, 2 m \pi)$, where $n, m \in \mathbb{Z}$. These spectral features are well understood for normal quasiparticle scattering with small $k$ values in addition to umklapp processes related to the two $\mathrm{Fe}$ atom unit cell periodicity. In that case, we obtain a spectral image in agreement with the apparent $C_{4}$ symmetry in the STM topography images. It seems that the interband scattering of the normal quasiparticles between the center of the BZ and the pockets around the $M$ points is not visible in our data. It could be that symmetry considerations forbid the related channels or that the spectral intensity is negligible with respect to the one of the umklapp processes.

The analysis of the scattering channels inside the superconducting gap gives specific characteristic wave vectors which contrast from the ones of the normal quasiparticles. The high density of defects provokes, on purpose, the enhancement of the scattering phenomena and reinforces the weak spectral signatures and, in particular, the following ones. First, it seems that the spectral signatures around the $\Gamma_{n, m}$ points become fuzzier and more extended as if additional interactions emerged. More importantly, Fig. 3 evidences additional scattering channels in the vicinity of the $(n \pi, m \pi)$ points. Only two characteristic wave vectors are found in the first Brillouin zone at $\boldsymbol{q}_{1}^{1 \mathrm{st} \mathrm{BZ}} \approx \frac{5}{6}(-\pi,+\pi)$ and $\boldsymbol{q}_{2}^{1 \mathrm{st} \mathrm{BZ}} \approx \frac{5}{6}(+\pi,-\pi)$. Let us stress that a $C_{4}$ symmetry would imply the same signatures at conjugated wave vectors $g^{ \pm} \approx \frac{5}{6} \mp(\pi, \pi)$. This is not the case and the scattering of the Bogoliubov quasiparticles in the reciprocal space has a $C_{2}$ symmetry. In the second Brillouin zones, four more channels are visible at the following wave vectors: $\boldsymbol{q}_{1}^{2 \text { nd BZ }} \approx(\pi, \pi)+\frac{5}{6}(-\pi, \pi), q_{2}^{2 \text { nd } \mathrm{BZ}} \approx$ $(\pi, \pi)+\frac{5}{6}(\pi,-\pi), \quad \boldsymbol{q}_{3}^{2 \mathrm{nd} \mathrm{BZ}} \approx(-\pi,-\pi)+\frac{5}{6}(-\pi, \pi)$, and $\boldsymbol{q}_{4}^{2 \text { nd BZ }} \approx(-\pi,-\pi)+\frac{5}{6}(\pi,-\pi)$. Only two of the channels in the third BZs are visible (the other ones are too far in the reciprocal space to be recorded) at wave vectors 
(a)

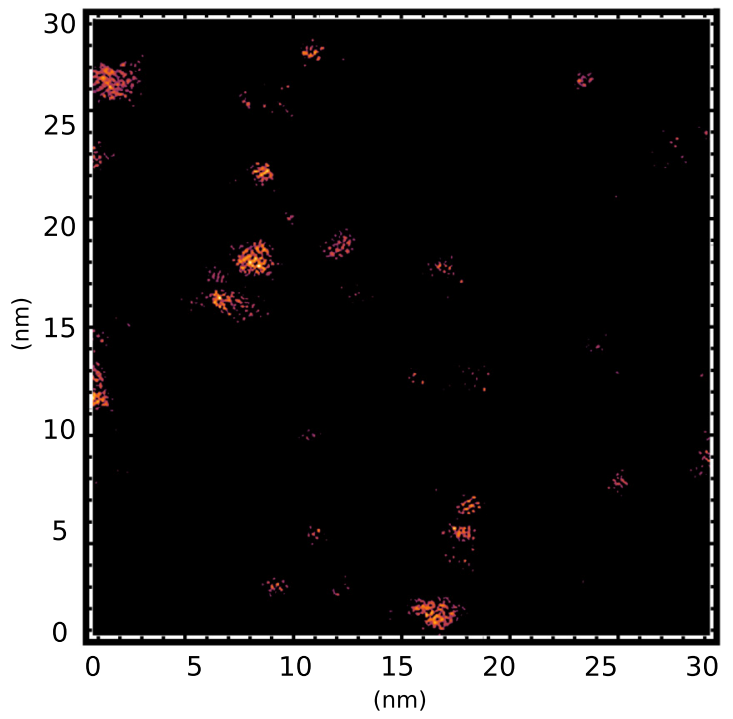

(g)

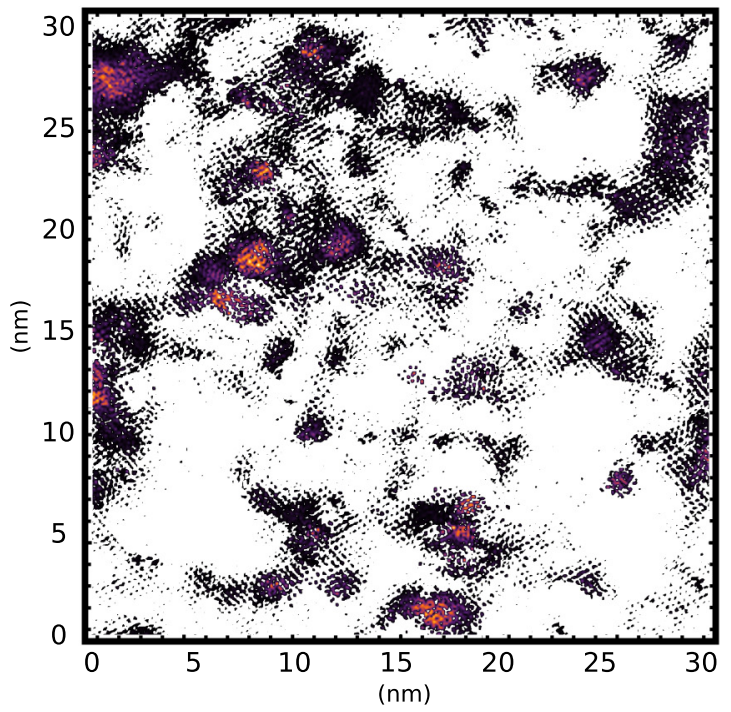

(b)

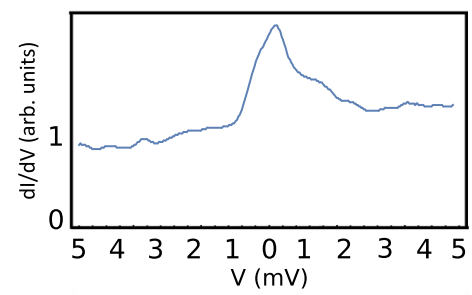

(c)

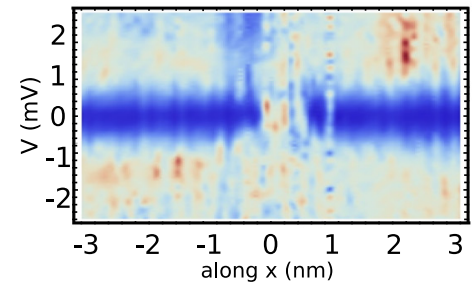

(d)

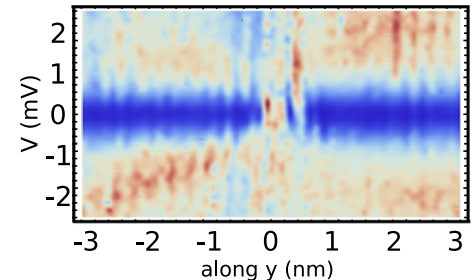

(e)

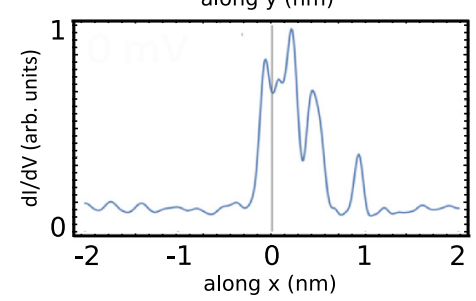

$(f)$

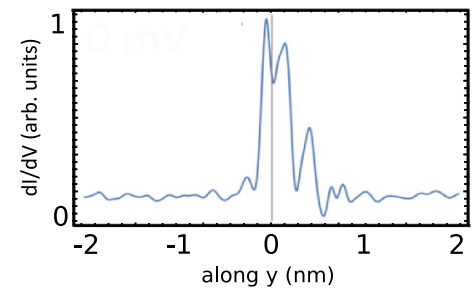

FIG. 2. Defect analysis. (a) Mapping of the locations where superconductivity is broken (the superconducting gap is filled). (b) Average spectrum corresponding to these locations. These defects are similar to Yu-Shiba-Rusinov impurities in a standard superconductor. (c), (d) Analysis of the tunneling conductance around these impurities as a function of energy and distance along the $\boldsymbol{a}$ and $\boldsymbol{b}$ axis. (e), (f) Spectral intensity at zero bias around these impurities as a function of distance along $\boldsymbol{a}$ and $\boldsymbol{b}$. (g) Mapping of the location where superconductivity is weakened (where the in-gap spectral weight is superior to the spatial average of the spectral weight), i.e., where the gap is weakened but not completely filled. Stabilization parameters: $I=600 \mathrm{pA}, V=8 \mathrm{mV}$.

$\boldsymbol{q}_{1}^{3 \mathrm{rd} \mathrm{BZ}} \approx \frac{7}{6}(-\pi, \pi)$ and $\boldsymbol{q}_{2}^{3 \mathrm{rd} \mathrm{BZ}} \approx \frac{7}{6}(\pi,-\pi)$. All these signatures are in agreement with $C_{2}$ symmetry.

The experimental data tend to show that the spectral density of the additional scattering channels depends on the considered index of the Brillouin zone. For example, the signal is quite asymmetric with respect to $M^{+,+}=(+\pi,+\pi)$ along the $\Gamma_{10} \Gamma_{01}$ direction, whereas the two spectral signatures are both located in second-order Brillouin zones. A composition of two excitations with $\boldsymbol{q}_{1,2}^{1 \mathrm{st} B Z}$ with or without umklapp processes could also be at the origin of the broadening of the spectral features at the $\Gamma$ points. The additional spectral signatures subsist in a narrow energy range $(|E|<0.9 \mathrm{meV})$ inside the superconducting gap whereas they do not exist outside the gap. The crossover from $C_{2}$ to $C_{4}$ symmetry when exploring energies in and out of the gap strongly suggests that the $C_{2}$ symmetry is inherent to the superconducting physics and is not related to the geometry of the STM experiment where the probe is necessarily outside of the crystal of $S_{4}$ symmetry. So, it reveals a hidden $C_{2}$ character of nesting vectors which are relevant for the Cooper pairs (i.e., for the Bogoliubov quasiparticles) and not for the "normal" quasiparticles.

These channels could be related to nesting vectors from the hole bands at the center of the Brillouin zone towards the electron pockets around $M$. The spectral weight of these channels being anisotropic around the $M$ points indicates either an anisotropic superconducting gap or an anisotropic susceptibility for the pair breaking, as expected for an interaction with nematic order excitations. Our STS data [Figs. 2(i)-2(1)] did not show any observable energy dispersion of the discovered scattering channels. This observation could point towards a 
(a)

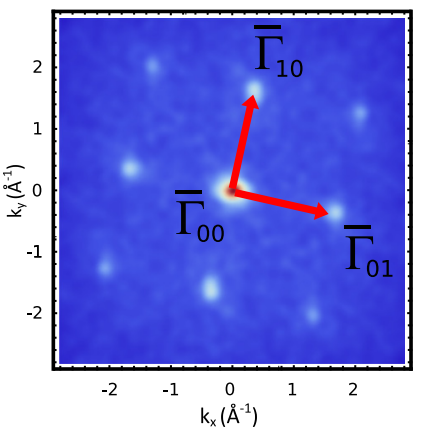

(d)

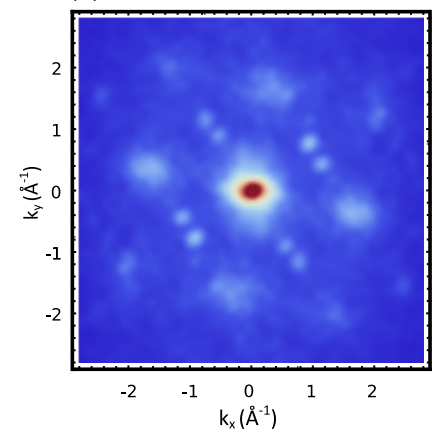

(i)

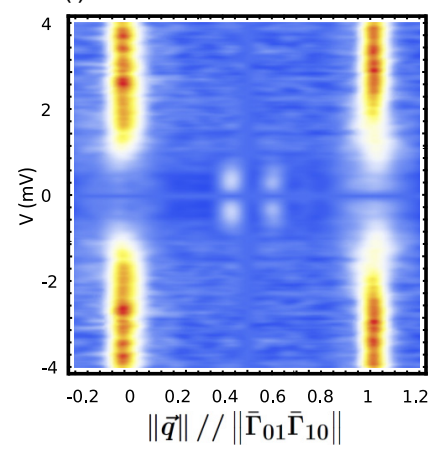

(b)

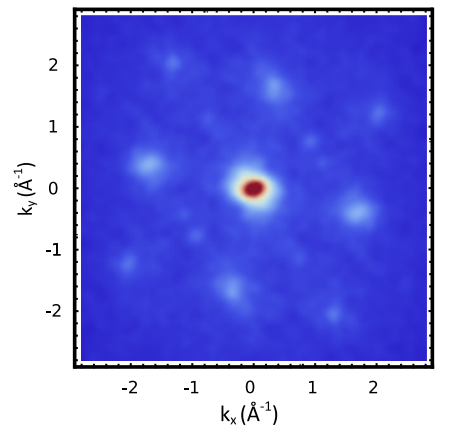

(e)

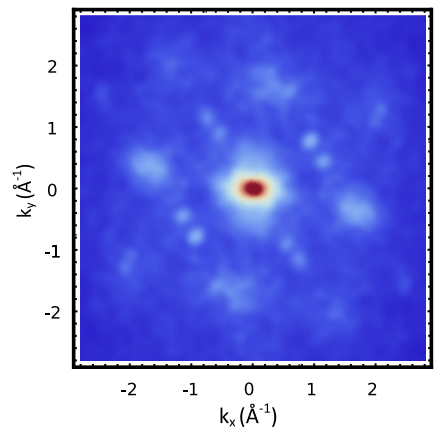

(j)

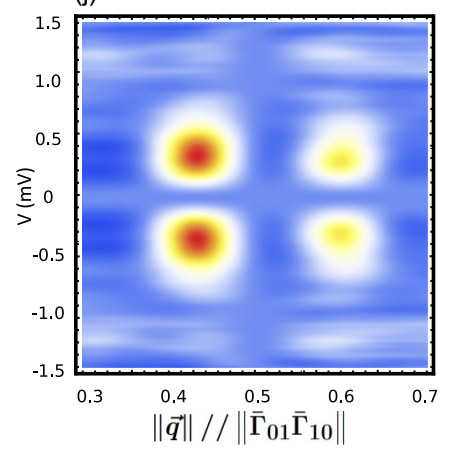

(c)

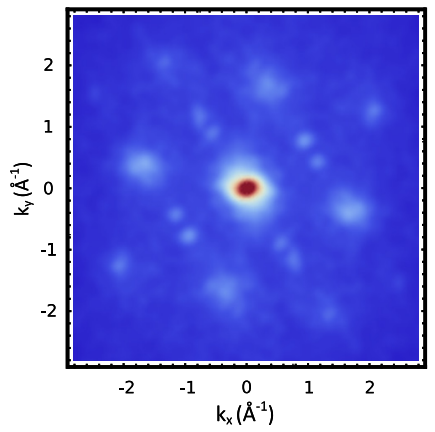

(f)

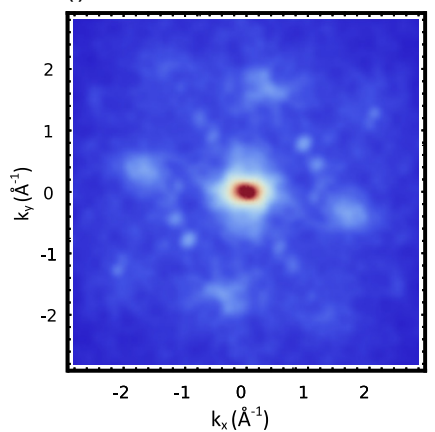

(k)

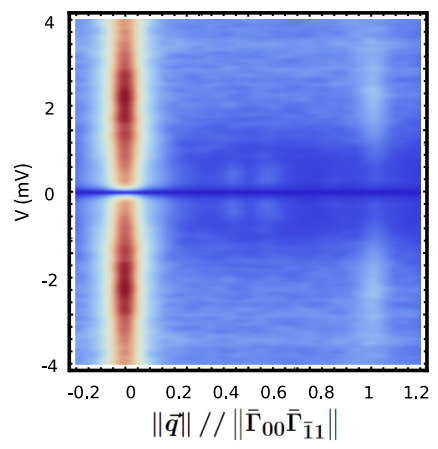

(g)

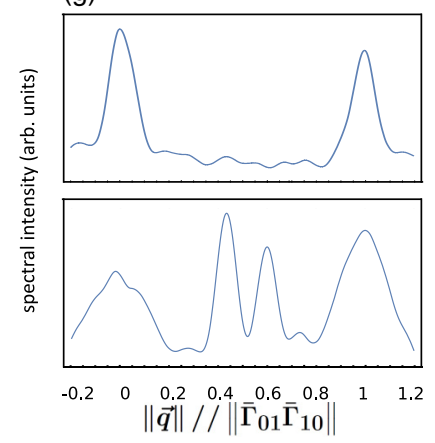

(h)

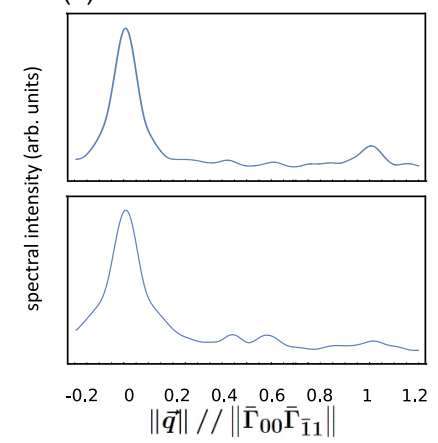

(I)

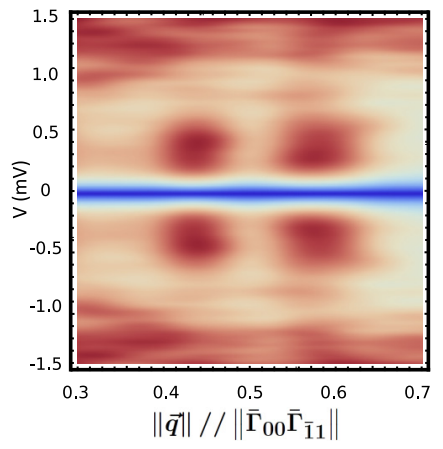

FIG. 3. (a)-(f) Fourier transform of $\pm V$ antisymmetrized conductance images at absolute voltage $(2,0.8,0.6,0.4,0.2$, and $0 \mathrm{mV})$, respectively (see Supplemental Material [37] for more details). (b)-(f) correspond to energies inside the superconducting gap. (g) Profiles of the spectral intensity along $\Gamma_{10} M^{+,+} \Gamma_{01}$ at $2 \mathrm{mV}$ (top) and $0.4 \mathrm{mV}$ (bottom). (h) Profiles of the spectral intensity along $\Gamma_{00} M^{-,+} \Gamma_{\overline{1} 0}$ at 2 $\mathrm{mV}$ (top) and $0.4 \mathrm{mV}$ (bottom). (i), (j) Voltage dependance of the spectral intensity along $\Gamma_{10} M^{+,+} \Gamma_{01}$. The two channels are separated from each other by $\approx(0.17 \pm 0.01) \Gamma_{10} \Gamma_{01}$. (k), (l) Voltage dependance of the spectral intensity along $\Gamma_{00} M^{-,+} \Gamma_{\overline{1} 0} . k x$ and $k y$ refer to (fast and slow) scan directions in real space. Stabilization parameters: $I=300 \mathrm{pA}, V=6 \mathrm{mV}$.

different origin than the nodes in the order parameter. These channels are not related to a simple spin fluctuation process because they exist at an energy scale well below the energy of the magnetic modes discovered by neutron scattering [14] (above $2.5 \mathrm{meV}$ ) and their spectral signatures are located slightly off the antiferromagnetic nesting wave vectors. In conclusion, we attribute the $C_{2}$ symmetry and the lack of dispersion of these scattering channels to the interaction of the Bogoliubov quasiparticle with the nematic order. All these experimental features are consistent with a scenario where the nematic order competes with the superconductivity. The discovery of only one orientation for the $C_{2}$ symmetry in the spectroscopic data even if the sample exhibits both orthorhombic orientational domains is somehow surprising and would support the fact that structure and nematicity are not necessarily locked together. However, the ratio between the two orthorhombic orientational domains is not determined and one structural domain could be strongly dominant. In that case the nematic character of this dominant domain could dictate the symmetry of the fast Fourier transform (FFT) analysis, the spectral intensity for small domains in the real space being spread out in the reciprocal space.

We thank Luca de' Medici and Benoit Fauqué for helpful discussions. S.P. and D.R. acknowledge C'NANO Ile-deFrance, DIM NanoK, for the support of the Nanospecs project. The Ph.D. grant of J.B. is supported by the Labex Matisse and the Nexans Chair through the PhaseOnSi project. We thank the French National Research Agency for the support of the SUPERSTRIPES project, No. ANR-15-CE30-0026. P.T. acknowledges the financial support of UGA and Grenoble INP through the AGIR-2013 contract of S.K. 
[1] S. A. Kivelson, E. Fradkin, and V. J. Emery, Nature (London) 393, 550 (1998).

[2] A. E. Böhmer and A. Kreisel, J. Phys.: Condens. Matter 30, 023001 (2018).

[3] J. Zhao, D. T. Adroja, D.-X. Yao, R. Bewley, S. Li, X. F. Wang, G. Wu, X. H. Chen, J. Hu, and P. Dai, Nat. Phys. 5, 555 (2009)

[4] J.-H. Chu, J. G. Analytis, K. De Greve, P. L. McMahon, Z. Islam, Y. Yamamoto, and I. R. Fisher, Science 329, 824 (2010).

[5] T.-M. Chuang, M. P. Allan, J. Lee, Y. Xie, N. Ni, S. L. Bud'ko, G. S. Boebinger, P. C. Canfield, and J. C. Davis, Science 327, 181 (2010).

[6] M. Yi, D. Lu, J.-H. Chu, J. G. Analytis, A. P. Sorini, A. F. Kemper, B. Moritz, S.-K. Mo, R. G. Moore, M. Hashimoto et al., Proc. Natl. Acad. Sci. U.S.A. 108, 6878 (2011).

[7] E. P. Rosenthal, E. F. Andrade, C. J. Arguello, R. M. Fernandes, L. Y. Xing, X. C. Wang, C. Q. Jin, A. J. Millis, and A. N. Pasupathy, Nat. Phys. 10, 225 (2014).

[8] F. Wand, S. A. Kivelson, and D.-H. Lee, Nat. Phys. 11, 959 (2015).

[9] R. M. Fernandes, A. V. Chubukov, and J. Schmalian, Nat. Phys. 10, 97 (2014).

[10] P. O. Sprau, A. Kostin, A. Kreisel, A. E. Böhmer, V. Taufour, P. C. Canfield, S. Mukherjee, P. J. Hirschfeld, B. M. Andersen, and J. C. S. Davis, Science 357, 75 (2017).

[11] S.-H. Baek, D. V. Efremov, J. M. Ok, J. S. Kim, J. van den Brink, and B. Büchner, Nat. Mater. 14, 210 (2014).

[12] K. Nakayama, Y. Miyata, G. N. Phan, T. Sato, Y. Tanabe, T. Urata, K. Tanigaki, and T. Takahashi, Phys. Rev. Lett. 113, 237001 (2014).

[13] A. E. Böhmer, T. Arai, F. Hardy, T. Hattori, T. Iye, T. Wolf, H. v. Löhneysen, K. Ishida, and C. Meingast, Phys. Rev. Lett. 114, 027001 (2015).

[14] Q. Wang, Y. Shen, B. Pan, Y. Hao, M. Ma, F. Zhou, P. Steffens, K. Schmalz, T. R. Forrest, M. Abdel-Hafiez et al., Nat. Mater. 15, 159 (2016).

[15] M. Ma, P. Bourges, Y. Sidis, Y. Xu, S. Li, B. Hu, J. Li, F. Wang, and Y. Li, Phys. Rev. X 7, 021025 (2017).

[16] P. Johnson, G. Xu, and W. Yin, Iron-Based Superconductivity (Springer, Basel, 2015).

[17] M. D. Watson, A. A. Haghighirad, L. C. Rhodes, M. Hoesch, and T. K. Kim, New J. Phys. 19, 103021 (2017).

[18] L. Fanfarillo, J. Mansart, P. Toulemonde, H. Cercellier, P. Le Fèvre, F. Bertran, B. Valenzuela, L. Benfatto, and V. Brouet, Phys. Rev. B 94, 155138 (2016).

[19] M. D. Watson, T. K. Kim, A. A. Haghighirad, N. R. Davies, A. McCollam, A. Narayanan, S. F. Blake, Y. L. Chen, S. Ghannadzadeh, A. J. Schofield et al., Phys. Rev. B 91, 155106 (2015).
[20] M. D. Watson, T. K. Kim, L. C. Rhodes, M. Eschrig, M. Hoesch, A. A. Haghighirad, and A. I. Coldea, Phys. Rev. B 94, 201107 (2016).

[21] M. D. Watson, A. A. Haghighirad, H. Takita, W. Mansuer, H. Iwasawa, E. F. Schwier, A. Ino, and M. Hoesch, J. Phys. Soc. Jpn. 86, 053703 (2017).

[22] S. V. Borisenko, D. V. Evtushinsky, Z.-H. Liu, I. Morozov, R. Kappenberger, S. Wurmehl, B. Büchner, A. N.Yaresko, T. K. Kim, M. Hoesch et al., Nat. Phys. 12, 311 (2016).

[23] P. Zhang, T. Qian, P. Richard, X. P. Wang, H. Miao, B. Q. Lv, B. B. Fu, T. Wolf, C. Meingast, X. X. Wu et al., Phys. Rev. B 91, 214503 (2015).

[24] M. Bendele, A. Ichsanow, Y. Pashkevich, L. Keller, T. Strässle, A. Gusev, E. Pomjakushina, K. Conder, R. Khasanov, and H. Keller, Phys. Rev. B 85, 064517 (2012).

[25] J. P. Sun, K. Matsuura, G. Z. Ye, Y. Mizukami, M. Shimozawa, K. Matsubayashi, M. Yamashita, T. Watashige, S. Kasahara, Y. Matsuda et al., Nat. Commun. 7, 12146 (2016).

[26] K. Kothapalli, A. E. Böhmer, W. T. Jayasekara, B. G. Ueland, P. Das, A. Sapkota, V. Taufour, Y. Xiao, E. Alp, S. L. Bud'ko et al., Nat. Commun. 7, 12728 (2016).

[27] C. Mann, arXiv:1509.07807.

[28] R. Wiesendanger, Scanning Probe Microscopy and Spectroscopy (Cambridge University Press, Cambridge, UK, 1994).

[29] A. Audouard, F. Duc, L. Drigo, P. Toulemonde, S. Karlsson, P. Strobel, and A. Sulpice, Europhys. Lett. 109, 27003 (2015).

[30] S. Karlsson, P. Strobel, A. Sulpice, C. Marcenat, M. Legendre, F. Gay, S. Pairis, O. Leynaud, and P. Toulemonde, Supercond. Sci. Technol. 28, 105009 (2015).

[31] P. Massat, D. Farina, I. Paul, S. Karlsson, P. Strobel, P. Toulemonde, M.-A. Méasson, M. Cazayous, A. Sacuto, S. Kasahara et al., Proc. Natl. Acad. Sci. U.S.A. 113, 9177 (2016).

[32] F.-C. Hsu, J.-Y. Luo, K.-W. Yeh, T.-K. Chen, T.-W. Huang, P. M. Wu, Y.-C. Lee, Y.-L. Huang, Y.-Y. Chu, D.-C. Yan et al., Proc. Natl. Acad. Sci. U.S.A. 105, 14262 (2008).

[33] D. Huang, T. A. Webb, C.-L. Song, C.-Z. Chang, J. S. Moodera, E. Kaxiras, and J. E. Hoffman, Nano Lett. 16, 4224 (2016).

[34] W. Qing-Yan, L. Zhi, Z. Wen-Hao, Z. Zuo-Cheng, Z. Jin-Song, L. Wei, D. Hao, O. Yun-Bo, D. Peng, C. Kai et al., Chin. Phys. Lett. 29, 037402 (2012).

[35] S. Kasahara, T. Watashige, T. Hanaguri, Y. Kohsaka, T. Yamashita, Y. Shimoyama, Y. Mizukami, R. Endo, H. Ikeda, K. Aoyama et al., Proc. Natl. Acad. Sci. U.S.A. 111, 16309 (2014).

[36] G. C. Menard, S. Guissart, C. Brun, S. Pons, V. S. Stolyarov, F. Debontridder, M. V. Leclerc, E. Janod, L. Cario, D. Roditchev et al., Nat. Phys. 11, 1013 (2015).

[37] See Supplemental Material at http://link.aps.org/supplemental/ 10.1103/PhysRevB.98.220502 for more information on conductance images before Fourier transformation and on the symmetrization procedure. 